\title{
ESTUDIO DE LAS REABSORCIONES RADICULARES ASOCIADAS A CANINOS RETENIDOS MAXILARES EN TOMOGRAFÍA CONE BEAM
}

Cone beam tomography study of root resorption associated with retained canines

Fecha de Recepción: 12 de febrero 2020
Estudo da tomografia por feixe cônico da reabsorção radicular associada a cães retidos

Aceptado para su publicación: 20 de abril 2020

\section{Autores: \\ Belén M. Tomas Arancibia Adriana N. Poletto}

1. Becaria EVC Resol. PN 389/18 CIN. Cátedra Periodoncia. Facultad de Odontología. Universidad Nacional de Cuyo. 2. Cátedra Diagnóstico por Imágenes I y II. Facultad de Odontología. Universidad Nacional de Cuyo.

Correspondencia: Adriana N. Poletto

Correo electrónico: dimagenes@fodonto.uncu.edu.ar

Conflicto de intereses: los autores declaran no tener conflictos de interés.

Fuente de financiamiento: Consejo Interuniversitario Nacional MECCYT RA (EVC Resol. PN 389/18 CIN).

\section{Resumen}

La alta frecuencia con la que se presentan casos de caninos impactados en el Servicio de Imagen Diagnóstica (SID) de la Facultad de Odontología de la Universidad Nacional de Cuyo (FO-UNCU), nos estimuló a detectar y evaluar las reabsorciones radiculares de los elementos dentarios adyacentes a dichos caninos. El propósito del presente estudio es identificar reabsorciones radiculares en elementos permanentes causadas por caninos maxilares retenidos. Las mediciones fueron tomadas en un software de visualización Roemexis viewerTM, que permite observar cada caso tomado por una tomografía de haz cónico o cone-beam. La muestra estuvo constituida por estudios tomográficos de haz cónico de pacientes derivados al SID para estudio de caninos impactado. Los resultados hallados muestran una prevalencia de reabsorciones radiculares vinculadas a caninos permanentes maxilares retenidos especialmente de incisivos laterales, centrales y en menor frecuencia premolares. La magnitud de estas reabsorciones son significativas y su estudio tomográfico permite detectarlas preventivamente. Los hallazgos constituyen un aporte a los datos epidemiológicos de la región de Cuyo, RA.

Palabras clave: caninos, impactados, reabsorciones, radiculares.

\footnotetext{
Abstract

The high frequency with that cases of impacted canines are presented in the Diagnostic Imaging Service (SID) of the Faculty of Dentistry of the National University of Cuyo (FO-UNCU), decided us to detect and evaluate the root resorption of adjacent dental elements of those canines. The purpose of the present study is to identify root resorption in permanent elements cau-
} 
sed by retained maxillary canines. The measurements were taken in a Roemexis viewerTM visualization software, which allows observing each case taken by a cone-beam technic or cone-beam tomography. The sample consisted of cone beam tomographic studies of patients referred to the DIS for study of impacted canines. The results found showed a prevalence of root resorption associated with permanent maxillary canines retained especially from the lateral, central incisors and, less frequently, premolars. The magnitude of these reabsorptions are significant and their tomographic study allows them to be detected preventively. The findings constitute a contribution to the epidemiological data of the Cuyo region, RA.

Key words: canines, impacted, resorption, root.

\section{Resumo}

A alta frequência com que casos de caninos impactados são apresentados no Serviço de Diagnóstico por Imagem (SDI) da Faculdade de Odontologia da Universidade Nacional de Cuyo (FO-UNCU), nos motivou a detectar e avaliar a reabsorção radicular de elementos dentários adjacentes aos referidos caninos. $O$ objetivo do presente estudo é identificar a reabsorção radicular em elementos permanentes causados por caninos superiores retidos. As medições foram realizadas em um software de visualização Roemexis viewerTM, que permite observar cada caso realizado por uma tomografia de feixe cônico ou feixe cônico. A amostra foi constituída por estudos tomográficos de feixe cônico de pacientes encaminhados ao SDI para estudos caninos impactados. Os resultados encontrados mostram uma prevalência de reabsorção radicular ligada a caninos superiores permanentes, especialmente retidos de incisivos laterais, centrais e com menor frequência de pré-molares. Os resultados constituem uma contribuição para os dados epidemiológicos da região de Cuyo RA.

Palavras-chave: caninos, impactados, reabsorção, raíz.

\section{Introducción}

Partiendo desde el punto de normalidad, el pro- ceso de erupción del canino superior comienza con un trayecto horizontal de formación del germen dental. A medida que se produce la calcificación de las porciones apicales del mismo se orienta hacia su canal de erupción rectificando esta posición horizontal, proceso que se concluye totalmente cuando se produce el contacto con el ápice del incisivo lateral $^{1}$. Este normal desarrollo eruptivo en algunos casos se puede interrumpir generando inclusiones óseas llamadas impactaciones y reabsorciones de elementos dentarios vecinos. Se conoce como diente impactado aquellos que se han formado dentro de hueso pero que han fracasado en el proceso de erupción. Numerosos autores, en un intento de predecir estas posibles inclusiones y reabsorciones, han estudiado estas complicaciones eruptivas y sus consecuencias en la tomografía computarizada cone beam TCCB ${ }^{2-4}$.

El acceso a la TCCB influye notablemente en la toma de decisiones de los odontólogos sobre el manejo de la reabsorción radicular, fundamentalmente de los incisivos, debido a los caninos maxilares ectópicos, siendo una herramienta de excelencia ${ }^{3}$.

La impactación del canino permanente es un problema clínico que puede provocar una serie de secuelas que van desde la pérdida de espacio en el arco para su correcta erupción, hasta la reabsorción radicular de dientes vecinos, el que es un fenómeno bien reconocido de ocurrencia en una proporción sorprendentemente alta de pacientes con caninos impactados ${ }^{5}$. La impactación y erupción ectópica de caninos representa un gran problema por la ausencia de síntomas. A veces puede percibirse a través de la persistencia más allá de la edad cronológica de erupción de los elementos dentarios temporarios o ya exfoliados, la ausencia en boca de los sucesores permanentes.

Cualquier pieza dentaria puede encontrarse impactada pero la mayor frecuencia de impactación se observa en terceros molares inferiores y superiores, seguidos por caninos superiores, segundo premolares superiores e inferiores y los incisivos centrales superiores. Los caninos no erupcionados ocurren con una frecuencia 20 veces mayor en el maxilar superior que en la mandíbula y casi siempre están rotados de 60 a 90 grados sobre su eje longitudinal ${ }^{5}$. 
La incidencia de impactación de caninos superiores varía entre $0,92 \%$ a $2,2 \%{ }^{6}$. Con relación al sexo son dos veces más común en mujeres $(1,17 \%)$ que en varones $(0,51 \%)$. La incidencia de impactación por palatino excede a la vestibular en una proporción de por lo menos 3:1. El 8\% de todas las impactaciones caninas ocurren bilateralmente ${ }^{7}$. La etiología de la impactación canina es multifactorial y difícil de definir. Rara vez ocurre de forma aislada ya que es común observarla acompañada con agenesia o anomalías en forma, de incisivos laterales, con diente deciduos retenidos, con dientes supernumerarios o con una deficiencia esquelética premaxilar (FLAP) 8 .

Hay que destacar que los caninos superiores están predispuestos a quedarse incluidos por presentar el período más largo de desarrollo, además de la vía de erupción más extensa y tortuosa9. Diversos factores locales y generales están relacionados con la impactación de caninos superiores: retención prolongada o pérdida prematura del canino primario, discrepancia dentoalveolar, posición anormal del germen dental, patología localizada como quistes, neoplasias, malformaciones, odontomas, supernumerarios, agenesia o alteración en la forma de incisivos laterales, presencia de fisura alveolar, anquilosis, trauma, origen idiopático, iatrogénico, aberración en la formación de la lámina dental, problemas nasales y respiratorios, variación en el tamaño de la raíz del diente, variación en el tiempo de formación radicular, secuencia de erupción anormal, exceso de espacio, cantidad de reabsorción del diente primario, forma de arco estrecha y herencia ${ }^{10}$.

Los resultados de diversos estudios indican que los dientes permanentes son susceptibles a tener reabsorción radicular aún en ausencia de factores sistémicos cuando cierta presión es aplicada a sus raíces. Ya que los dientes reabsorbidos no padecen dolor, los pacientes no detectan esta situación hasta que la extensión del daño es irreversible ${ }^{11,12}$. En orden de prevención de estos resultados, la detección temprana de estas retenciones de caninos es de vital importancia, de esta manera cuando existe falta de espacio para la erupción normal de los caninos permanentes, el examen radiográfico es esencial para establecer un diagnóstico y tratamiento adecuados. La extracción temprana de caninos o primeros pre- molares, o la tracción guiando el proceso eruptivo antes de que la reabsorción de los incisivos suceda es de vital importancia para la preservación de las piezas dentarias adyacentes. La reabsorción radicular de los incisivos laterales superiores causada por la erupción de los caninos es bien conocida y es un fenómeno relativamente común ${ }^{13}$. La introducción de la TCCB ha permitido diseñar nuevas estrategias diagnósticas y terapéuticas. Se ha demostrado cómo la adición de una tercera dimensión, visualizada en TCCB, a la información radiográfica puede alterar notablemente detección de reabsorciones radicales y las descripciones de esta prevalencia ${ }^{14}$.

La impactación canina maxilar aumenta el riesgo de reabsorción radicular de los dientes adyacentes, el cual es considerado un problema de proximidad física; dicha proximidad $(1 \mathrm{~mm})$ entre el canino impactado y una raíz adyacente es el elemento más importante que nos permite predecir la reabsorción radicular ${ }^{15}$. Dado que los elementos dentarios vecinos reabsorbidos en su mayoría se encuentran libres de dolor, la detección temprana mediante examen radiográfico es esencial para establecer un diagnóstico adecuado. La ubicación de los caninos permanentes maxilares (CPM) afectados varía enormemente en 3 planos y la reabsorción de los incisivos permanentes vecinos es común. Su detección precoz permite decidir cuál es el tratamiento más acorde para solucionar esa retención y las consecuencias producto de la misma ${ }^{16}$.

Los objetivos de este trabajo fueron: 1) detectar y tipificar las retenciones de CPM en estudios TCCB; 2) identificar las reabsorciones radiculares asociadas a CPM en estudios TCCB; 3) estratificar las reabsorciones de CPM en estudios TCCB.

\section{Materiales y Métodos}

Diseño de investigación El estudio realizado fue observacional correlacional en un corte transversal de tiempo 2018-2019. El diseño muestral fue por conglomerado (clúster) en el SID.

A fin de garantizar los derechos de los individuos, en el presente estudio no se identificaron datos personales preservando la identidad. 
INVESTIGACIÓN

\section{REFO}

VOL. XIII | No 1| AÑO 2020

ISSN 1668-7280

ISSN-E 2683-7986
El método utilizado se basa en el encuadre topográfico de las reabsorciones radiculares observadas y la medición del grado de afectación de las mismas en un software de reconstrucción tridimensional de tomógrafos de haz cónico en escala milimetrada ${ }^{17-}$ 20.

La detección de las reabsorciones fue medinate navegación en los planos axial y transaxial de los estudios TCCB. Una vez detectadas las reabsorciones, se procedió a medir las mismas con software RomexisTM (Figura 1).

Se realizó la observación de las reabsorciones en cortes transaxiales. Para realizar la medición de las mismas, se tuvo el siguiente criterio, si la reabsorción es unilateral como referencia métrica se utilizó como valor referencial las medidas en longitud y en ancho de la raíz del elemento homólogo en la arcada. En los casos, que las reabsorciones fueron bilaterales, las medidas de referencia aplicadas fueron las medidas anatómicas estándar del elemento dentario en cuestión, que aparecen en la literatura consultada (Figura 2).
La metodología es de observación y registro de los casos, en los que se detectaron reabsorciones radiculares de los elementos dentarios erupcionados, ocasionadas por elementos dentarios en proceso de erupción o en los cuales su erupción ha sido interrumpida (retenidos). Las mediciones se realizaron en casos, que constituyen el banco de datos del SID de la FO-UNCU, Mendoza, Argentina.

Se establecieron los datos de la frecuencia y prevalencia de la retención de elementos dentarios por alteraciones de su eje eruptivo.

\section{Resultados}

Se analizaron 115 casos del SID (FO-UNCU) de los cuales, 6 no presentaron retención y fueron desestimados. Un total de 109 estudios tomográficos de haz cónico, fueron analizados y categorizados. Se estudiaron 152 casos de CPM impactados.

La distribución por sexos arrojó que el 66 \% resultaron ser mujeres y el $34 \%$ hombres (Figura 3 ). De la muestra analizada, 43 de esos casos resultaron ser caninos retenidos bilaterales.

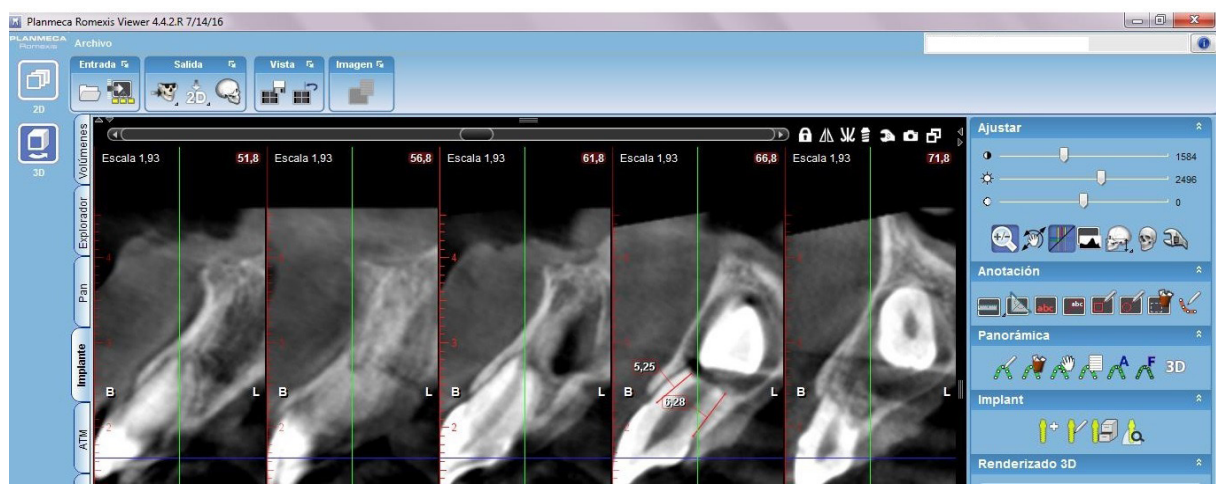

Figura 1. Medición de las reabsorciones con software.

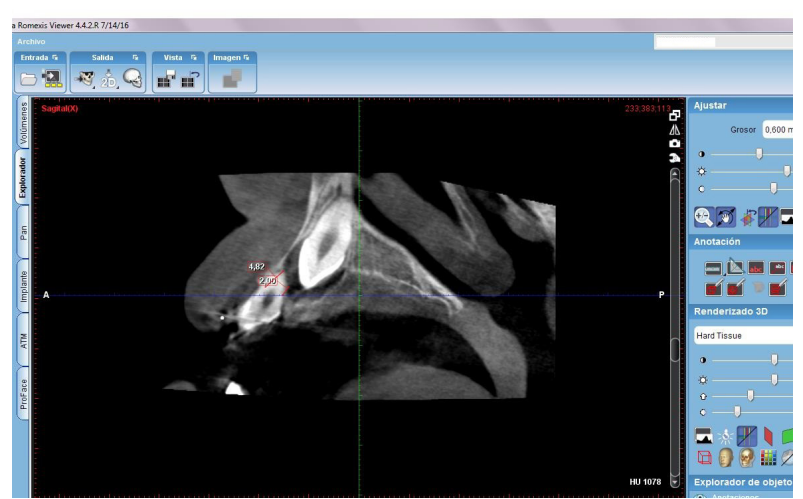

Figura 2. Muestra un caso de reabsorción radicular en cortes transaxiales

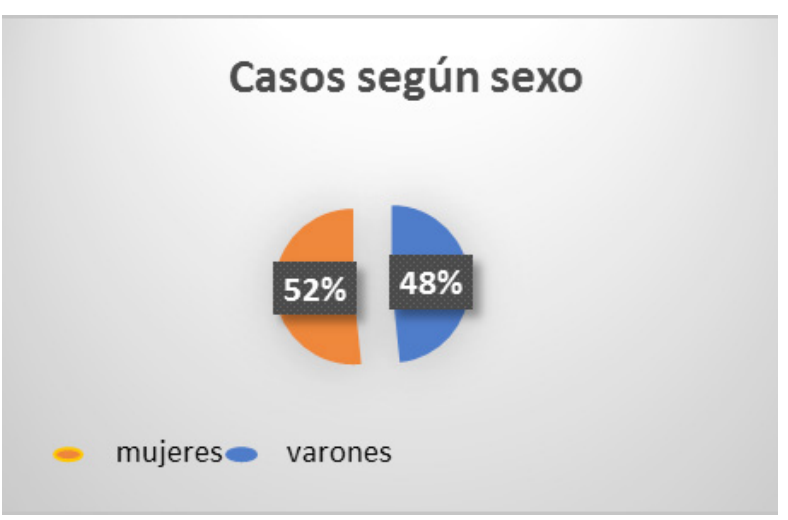

Figura 3. Distribución muestral por sexo. 
En total, se evaluaron 152 caninos impactados la distribución posicional de los caninos fue: 69 (48\%) casos de ubicación vestibular, $80(51,5 \%)$ casos de ubicación palatina y 3 casos de ubicación horizontal en los que no se pudo determinar la posición vestíbulo-palatina (Figura 4).

Se detectaron 39 casos de elementos dentarios con reabsorciones radiculares, asociadas a caninos retenidos, lo que constituye el $25,65 \%$ de los casos estudiados.

La distribución según elementos dentarios afectados fue: incisivos laterales $21,05 \%$, incisivos centrales $4,57 \%$ y premolares $0,06 \%$ (Figura 5 ).

En la Tabla I, se observa la distribución de elementos dentarios afectados (nomeclatura dentaria binaria). La reabsorción radicular se cuantificó. Los

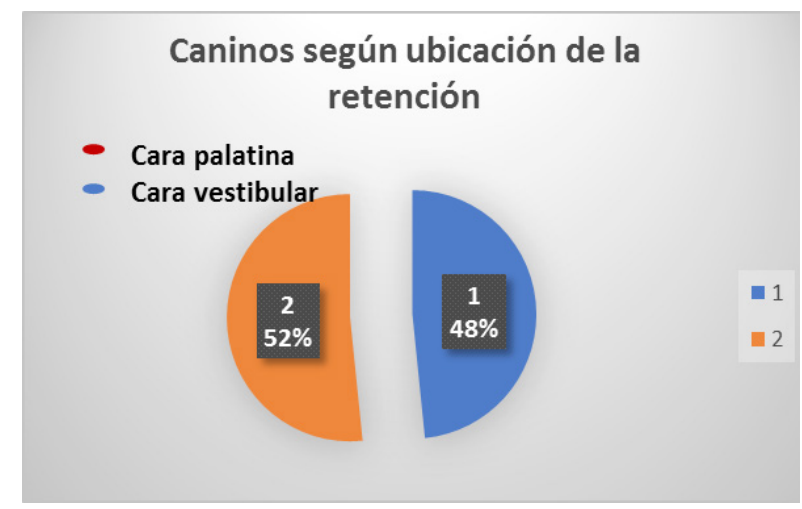

Figura 4. Distribución muestral según ubicación de retención. hallazgos fueron estratificados en milímetros y se observan en la Tabla II.

\section{Discusión}

Al usar la TCCB para estudiar el folículo dental, se pudo evaluar en 3 dimensiones. El folículo dental del canino maxilar en erupción varió en forma y ancho de 0,5 $\mathrm{mm}$ a $7 \mathrm{~mm}$ con un valor medio de 2,9 $\mathrm{mm}$, medido como la mayor distancia desde la corona del canino a la periferia del folículo. Esto significa que en algunos individuos, el ancho del folículo excedió los valores medios sustancialmente y excedió el ancho normal en aproximadamente, lo que indica que algunos folículos habían sufrido una transformación quística ${ }^{21}$.

Entre las impactaciones de los caninos, el $45.2 \%$ fueron impactadas bucal-labialmente, el $40.5 \%$

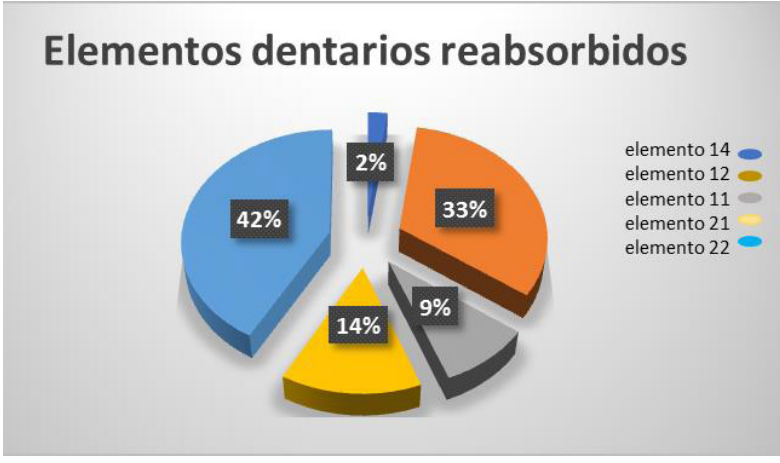

Figura 5. Distribución de reabsorciones por elementos dentarios.

Tabla I. Distribución de elementos dentarios afectados

\begin{tabular}{c|c|c|c|c}
\hline ELEMENTO & ELEMENTO & ELEMENTO & ELEMENTO & ELEMENTO \\
14 & 12 & 11 & 21 & 22 \\
1 caso & 14 casos & 4 casos & 6 casos & 18 casos \\
\hline
\end{tabular}

Tabla II. Reabsorción radicular estratificada en milímetros

\begin{tabular}{|c|c|c|c|c|}
\hline $\begin{array}{l}\text { REABSORCIÓN } \\
\text { EXPRESADA EN MM }\end{array}$ & $1 \mathrm{~mm}$ & $2 \mathrm{~mm}$ & $3 \mathrm{~mm}$ & $4 \mathrm{~mm}$ \\
\hline $\begin{array}{l}\text { CANTIDAD DE } \\
\text { ELEMENTOS } \\
\text { DENTARIOS }\end{array}$ & 21 & 9 & 5 & 4 \\
\hline
\end{tabular}


20

INVESTIGACIÓN
Revista de la Facultad de Odontología

Tomas Arancibia | Poletto

http://dx.doi.org/10.30972/rfo.1314336
REFO

VOL. XIII | N 1| AÑO 2020

ISSN 1668-7280

ISSN-E 2683-7986 fueron impactadas palatinamente y el $14.3 \%$ en el alvéolo medio. Estos valores reportados por la literatura internacional ${ }^{22,23}$, son similares a los encontrados en la presente investigación.

Estudios han determinado, que la reabsorción radicular estuvo presente en el $27.2 \%$ de los incisivos laterales y el $23.4 \%$ de los incisivos centrales, y el $94.3 \%$ de estas reabsorciones ocurrieron donde los caninos impactados estaban en contacto cercano con los incisivos ${ }^{23,24}$, valores similares a los hallados en la presente investigación.

Los resultados porcentuales, hallados en el presente trabajo referidos a impactaciones de CPM, son coincidentes con los hallazgos de diferentes muestras estudiadas 17-19,24. Los reportes, indican que las impactaciones y los cambios de los ejes normales eruptivos de los caninos permanentes superiores causan reabsorciones externas de dientes vecinos ${ }^{18,19}$, tal como lo indican los resultados de la presente investigación.

No se encontraron asociaciones entre la forma o el ancho del canino y el sexo, la edad o la etapa de erupción. Tampoco se observaron diferencias en el ancho o la forma entre los folículos ubicados dentro de una cripta ósea intacta y los folículos de los caninos que se acercan a la erupción en la cavidad oral $^{22}$. A este respecto, es importante documentar la posición canina mediante pruebas de palpación $y$, si es necesario, estudios tomográficos, durante las edades de 9-11 años. La presencia del canino temporario, en pacientes de 12 años de edad, es un buen indicador de que el canino permanente podría verse afectado ${ }^{23}$.

La metodología aplicada, para la estimación del grado de reabsorción radicular en el presente estudio no ha sido reportada en la bibliografía consultada, constituyendo, potencialmente, una herramienta de evaluación referencial en futuras investigaciones.

\section{Conclusiones}

El diagnóstico temprano y certero de reabsorciones radiculares, asociadas a un elemento permanente impactado, permite elegir el tratamiento correcto y efectuarlo lo antes posible, ya sea quirúrgico u ortodóntico, para brindarle al paciente la mejor solución a su problema y pronosticar efectivamente el estado del elemento dentario reabsorbido, eligiendo la mejor alternativa para el mismo.

El recurso que nos brinda la odontología digital, a partir del cual permite la evaluación de los casos que lo requieren, es infinito y muchas veces no es correctamente utilizado. La tomografía de haz cónico, debe ser un examen de elección cuando se sospecha de una posible inclusión canina, para abordar el diagnóstico y elegir el tratamiento de forma más precisa. La medición de las reabsorciones radiculares asociadas a la inclusión, nos permite predecir el pronóstico de la pieza y actuar en consecuencia.

\section{Referencias Bibliográficas}

1. Bjerklin K, Bondemark L. Ectopic maxillary canines and root resorption of adjacent incisors. Does computed tomography (CT) influence decision-making by orthodontists? Swed Dent J. 2008;32(4):179-85.

2. Alqerban $A 1$, Jacobs R, Lambrechts P, Loozen G, Willems G. Root resorption of the maxillary lateral incisor caused by impacted canine: a literature review. Clin Oral Investig. 2009;13(3):247-55. doi: 10.1007/s00784-0090262-8

3. Becker A1, Chaushu S. Long-term follow-up of severely resorbed maxillary incisors after resolution of an etiologically associated impacted canine. Am J Orthod Dentofacial Orthop. 2005;127(6):650-4. doi: 10.1016/j. ajodo.2004.03.031

4. Milberg DJ1. Labially impacted maxillary canines causing severe root resorption of maxillary central incisors. Angle Orthod. 2006;76(1):173-6. doi: 10.1043/0003-3219(2006)076[0173:LIMCCS]2.0.CO;2

5. Quereshy FA, Savell TA, Palomo JM. Applications of cone beam computed tomography in the practice of oral and maxillofacial surgery. J Oral Maxillofacial Surg. 2008; 66(4):791-6. doi:10.1016/j.joms.2007.11.018

6. Isman Ö, Yilmaz HH, Aktan AM, Yilmaz B. Indication for cone beam computed tomography in children and young patients in a Turkish subpopulation. Int J Paediatr Dent. 2017;27(3):183-190. doi: 10.1111/ipd.12250

7. Haney E, Gansky SA, Lee JS, Johnson E, Maki K, Miller AJ, Huang JC. Comparative analysis of traditional radiographs and cone-beam computed tomography volumetric images in the diagnosis and treatment planning of maxi- 
llary impacted canines. Am J Orthod Dentofac Orthop. 2010;137(5):590-7. doi: 10.1016/j.ajodo.2008.06.035

8. Botticelli S, Verna C, Cattaneo PM, Heidmann J, Melsen B. Two-versus three-dimensional imaging in subjects with unerupted maxillary canines. Eur J Orthod. 2011;33(4):344-9. doi: 10.1093/ejo/cjq102

9. Algerban A, Jacobs R, Fieuws S, Willems G. Comparison of two cone beam computed tomographic systems versus panoramic imaging for localization of impacted maxillary canines and detection of root resorption. Eur J Orthod. 2011;33(1):93-102. doi: 10.1093/ejo/cjq034

10. Lai CS, Bornstein MM, Mock L, Heuberger BM, Dietrich T, Katsaros C. Impacted maxillary canines and root resorptions of neighbouring teeth: a radiographic analysis using cone-beam computed tomography. Eur J Orthod. 2013;35(4):529-38. doi: 10.1093/ejo/cjs037

11. Bishara SE, Comer DD, McNeil MH, Montagano LN, Oesterle LJ, Yoongquist HW. Management of impacted canines. Am J. Orthod. 2010;69(4):371-87.

12. Rimes RJ, Mitchell CN, Willmot DR. Maxillary incisor root resorption in relation to the ectopic canine: a review of 26 patient. Eur J Orthod. 2017;19(1):79-84. doi: 10.1093/ejo/19.1.79

13. Ericson S, Kurol J. Radiographic assessment of maxiIlary canine eruption in children with clinical signs of eruption disturbances. Eur J Orthod. 1988;8(3):133-40.

14. Fournier A, Turcotte JY, Bernard C. Orthodontic considerations in the treatment of maxillary impacted canines. Am J Orthod. 2010;81(3):236-9.

15. Becker A, Chaushu S. Etiology of maxillary canine impaction: a review. Am J Orthod Dentofacial Orthop. 2015;148(4):557-67. doi: 10.1016/j.ajodo.2015.06.013
16. Jacoby $\mathrm{H}$. The etiology of maxillary canine impactions. Am J Orthod 2012;84(2):125-32.

17. Oberoi S, Knueppel S. Three-dimensional assessment of impacted canines and root resorption using cone beam computed tomography. Oral Surg Oral Med Oral Pathol Oral Radiol. 2012;113(2):260-7. doi: 10.1016/j. tripleo.2011.03.035

18. Scarfe WC, Farman AG, Sukovic P. Clinical applications of cone-beam computed tomography in dental practice. J Can Dent Assoc. 2006;72(1):75-80.

19. Nair MK, Nair UP. Digital and advanced imaging in endodontics: a review. J Endod. 2007;33(1):1-6.

20. Patel S, Dawood A, Mannocci F, Wilson R, Pitt Ford T. Detection of periapical bone defects in human jaws using cone beam computed tomography and intraoral radiography. Int Endod J. 2009;42(6):507-15.

21. Liu DG, Zhang WL, Zhang ZY, Wu YT, Ma XC. Localization of impacted maxillary canines and observation of adjacent incisor resorption with cone-beam computed tomography. Oral Surg Oral Med Oral Pathol Oral Radiol Endod. 2008;105(1):91-98. doi: 10.1016/j.tripleo.2007.01.030

22. Dalessandri D, Parrini S, Rubiano R, Gallone D, Migliorati M. Impacted and transmigrant mandibular canines incidence, aetiology, and treatment: a systematic review. Eur J Orthod. 2017;39(2):161-169. doi: 10.1093/ejo/ cjw027

23. Ericson S, Bjerklin $\mathrm{K}$. The dental follicle in normally and ectopically erupting maxillary canines. A computerized tomography study. Angle Orthod. 2001;71(5):332342. doi: 10.1043/0003-3219(2001)071<0333:TDFI$\mathrm{NA}>2.0 . \mathrm{CO} ; 2$ 